\title{
Cavernoma raquídeo epidural en reloj de arena. Presentación de un caso y revisión de la literatura
}

\author{
S. Iglesias; J. Ayerbe; J.L. Sarasa*; P. Sousa; C. Torres y P. Ruiz-Barnés
}

Servicio de Neurocirugía y *Servicio de Anatomía Patológica. Fundación Jiménez Díaz. Madrid.

\section{Resumen}

Los cavernomas raquídeos epidurales son malformaciones vasculares muy poco frecuentes que, excepcionalmente, pueden adquirir una morfología en "reloj de arena". En estos casos, el diagnóstico preoperatorio es habitualmente erróneo debido a su similitud clínicoradiológica con los tumores de la vaina nerviosa. Con el tratamiento quirúrgico se puede realizar una extirpación completa de estas lesiones sin apenas morbimortalidad, $y$ conseguir en consecuencia un excelente pronóstico.

Se presenta el caso de una mujer de 57 años a quien se le extirpó quirúrgicamente un cavernoma epidural en reloj de arena localizado en los segmentos C7 y D1. Se discuten las peculiaridades diagnósticas de este tipo de lesiones y se revisan las opciones terapéuticas disponibles en la actualidad.

PALABRAS CLAVE: Cavernoma. Angioma cavernoso. Epidural. Reloj de arena.

Dumbbell-shaped spinal epidural cavernous angioma. Case report and review of the literature

\section{Summary}

Spinal epidural cavernous angiomas are rare vascular malformations that exceptionally present with dumbbell-shape morphology. When it happens, preoperative misdiagnosis is the rule, because the clinicoradiological picture is quite similar to the nerve sheath tumours one. Spinal epidural cavernomas complete resection can be achieved with surgical treatment and scarcely morbi-mortality, and excellent outcome can be expected. We report a case of a 57 year-old woman carrying a dumbbell-shaped epidural cavernoma located at C7 and D1 levels that was surgically removed. Special diagnostic features of this kind of lesions are discussed

Recibido: 17-06-07. Aceptado: 13-06-07 and treatment options currently available are reviewed.

KEY WORDS: Cavernoma. Cavernous angioma. Epidural. Dumbbell-shaped.

Introducción

Los cavernomas, malformaciones cavernosas o hemangiomas cavernosos, son malformaciones vasculares que pueden localizarse en diversos órganos de la anatomía. Los de localización raquimedular, excluidos los hemangiomas cavernosos de cuerpo vertebral, son poco frecuentes y, dentro de este grupo, los cavernomas del espacio epidural representan menos del $4 \%$ de todas las lesiones expansivas epidurales $3,7,8,13,22-24,28,31$. Algunos casos extremadamente raros de CRE presentan una morfología en reloj de arena por su forma de crecimiento en dos componentes (intra y extrarraquídeo) unidos por un tercero transforaminal. Estos tipos de CRE, casi siempre son diagnosticados preoperatoriamente de forma errónea como neurofibromas o schwanomas.

Se presenta el caso de una paciente intervenida quirúrgicamente de un CRE con morfología en reloj de arena y localizado en los segmentos C7 y D1. Se realiza una revisión bibliográfica del tema y se discuten especialmente sus aspectos diagnósticos y terapéuticos.

\section{Caso clínico}

Se trata de una mujer de 57 años de edad que consultó por dolor a lo largo del borde interno del miembro superior derecho, de varios meses de evolución, sin otros síntomas asociados. En la exploración neurológica destacó solamente una hipoestesia en territorio radicular C8 derecho. Se realizaron una radiografía simple cervical que no mostró hallazgos de interés, y una RM de columna cervical que mostró una lesión con efecto de masa, bien delimitada, de

Abreviaturas. CRE: cavernoma raquideo espinal. RM: resonancia magnética. SNC: sistema nervioso central. TC: tomografía computarizada. 


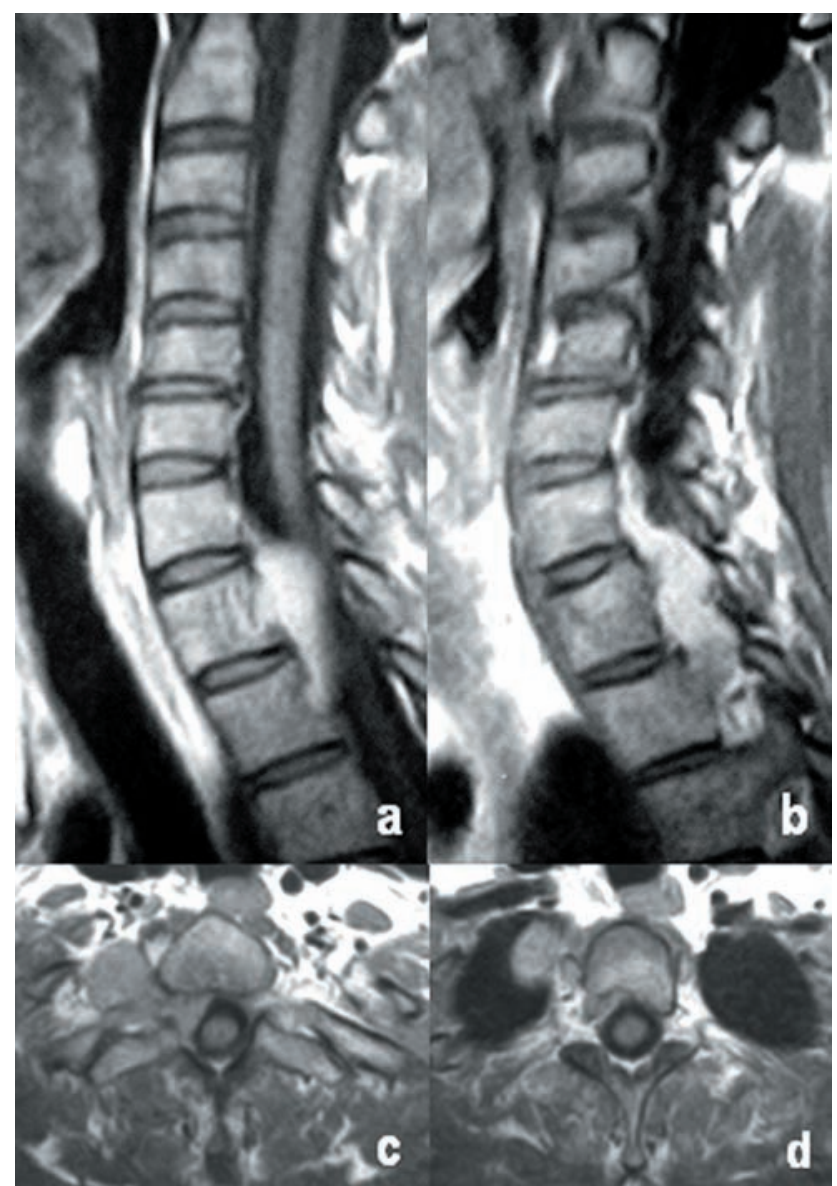

Figura 1. RM plano sagital T1 con contraste paramagnético que muestra la lesión bien delimitada con captación homogénea de gadolinio (a y b). RM plano axial T1 sin contraste en donde se observa el crecimiento transforaminal de la lesión "en reloj de arena” (c), y su componente intratorácico apical (d).

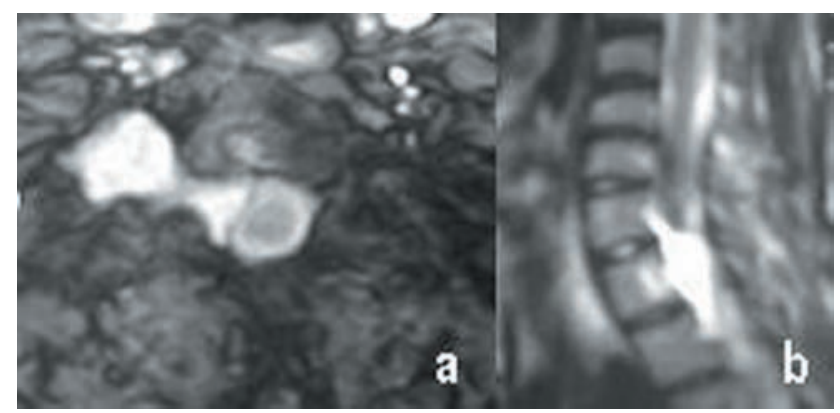

Figura 2. RM plano axial T2 (a) y sagital T2 (b) que muestran la lesión con señal homogéneamente hiperintensa.

bordes redondeados, localizada en niveles C7 y D1, y parcialmente en D2, y que ocupaba el espacio intrarraquídeo extramedular anterolateral derecho, con extensión al espacio intratorácico apical derecho a través del foramen intervertebral (morfología en "reloj de arena"). La lesión

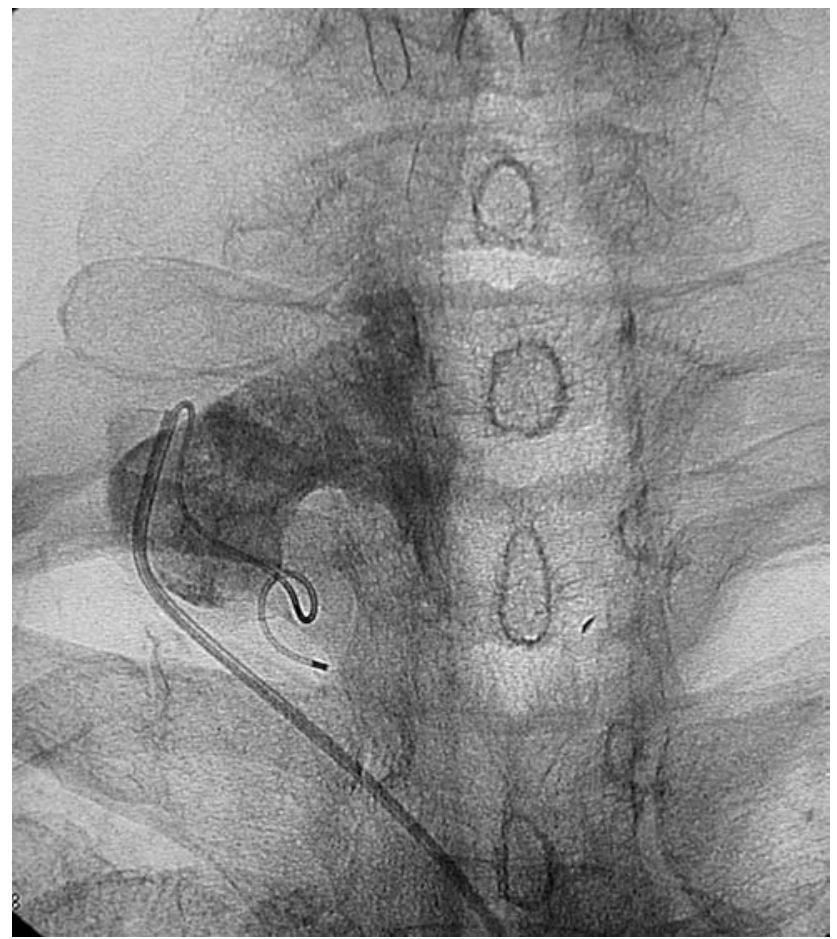

Figura 3. Cateterismo selectivo de la rama arterial aferente al cavernoma, a través de la cual se realizó la embolización preoperatoria.

era homogéneamente isointensa con respecto a los músculos adyacentes en la secuencia $\mathrm{T} 1$ de la RM, hiperintensa en T2, y captaba de forma homogénea e intensa el contraste paramagnético tras su administración intravenosa (Fig 1 y 2)

Ante la sospecha de un tumor hipervascularizado, se realizó una angiografía espinal que confirmó la presencia de una masa paravertebral derecha en niveles D1 y D2. El cateterismo selectivo de la única rama arterial aferente que tenía el tumor permitió su embolización endovascular sin incidencias (Fig 3).

Con el diagnóstico preoperatorio de neurinoma en "reloj de arena", se procedió a la resección quirúrgica de la lesión mediante una laminectomía cérvico-dorsal. Intraoperatoriamente se observó la presencia de una masa de aspecto angiomatoso, de localización epidural y con extensión extraforaminal, bien delimitada y con un buen plano de clivaje. Se realizó la extirpación completa de los fragmentos epidural y foraminal, quedando sin resecar el fragmento intratorácico. La embolización prequirúrgica del tumor facilitó su resección al disminuir las pérdidas sanguíneas intraoperatorias.

El diagnóstico anatomopatológico fue compatible con malformación vascular de estructura cavernosa bien delimitada por cápsula fibrosa (Fig 4).

La RM postquirúrgica mostró la extirpación completa 


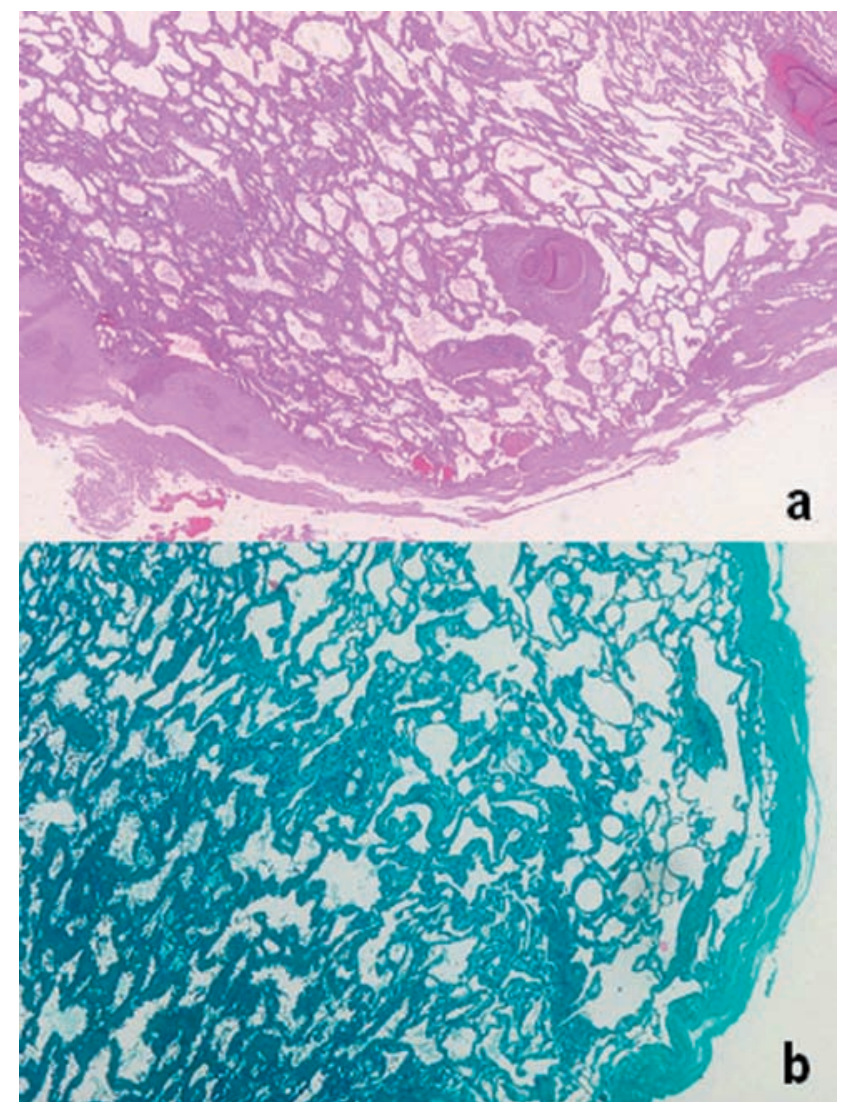

Figura 4. Anatomía microscópica de la malformación cavernosa. Preparación panorámica $H E$, en donde se observa material embólico en algunos espacios vasculares (a). Preparación Tricrómico de Masson, en donde se observan estructuras vasculares con paredes fibrosas comunes a luces contiguas (b).

del componente intrarraquídeo del cavernoma, persistiendo un resto intratorácico (Fig 5).

Durante el curso postoperatorio, la paciente presentó leves déficit motor y disestesias en territorio C8 derecho que permanecieron como únicas secuelas de la intervención. A lo largo de un seguimiento de 4 años, no se ha observado recidiva del cavernoma extirpado ni aumento de tamaño en el resto tumoral extrarraquídeo.

\section{Discusión}

Se considera que los cavernomas del SNC, denominados más correctamente como malformaciones cavernosas, son un tipo de malformación vascular desarrollado en las fases iniciales de la embriogénesis, con capacidad para aumentar progresivamente de tamaño a lo largo de la vida en algunos casos. Sin embargo, también se han descrito cavernomas desarrollados "de novo" en pacientes adultos, por lo que resulta difícil determinar si el origen de estas lesiones es

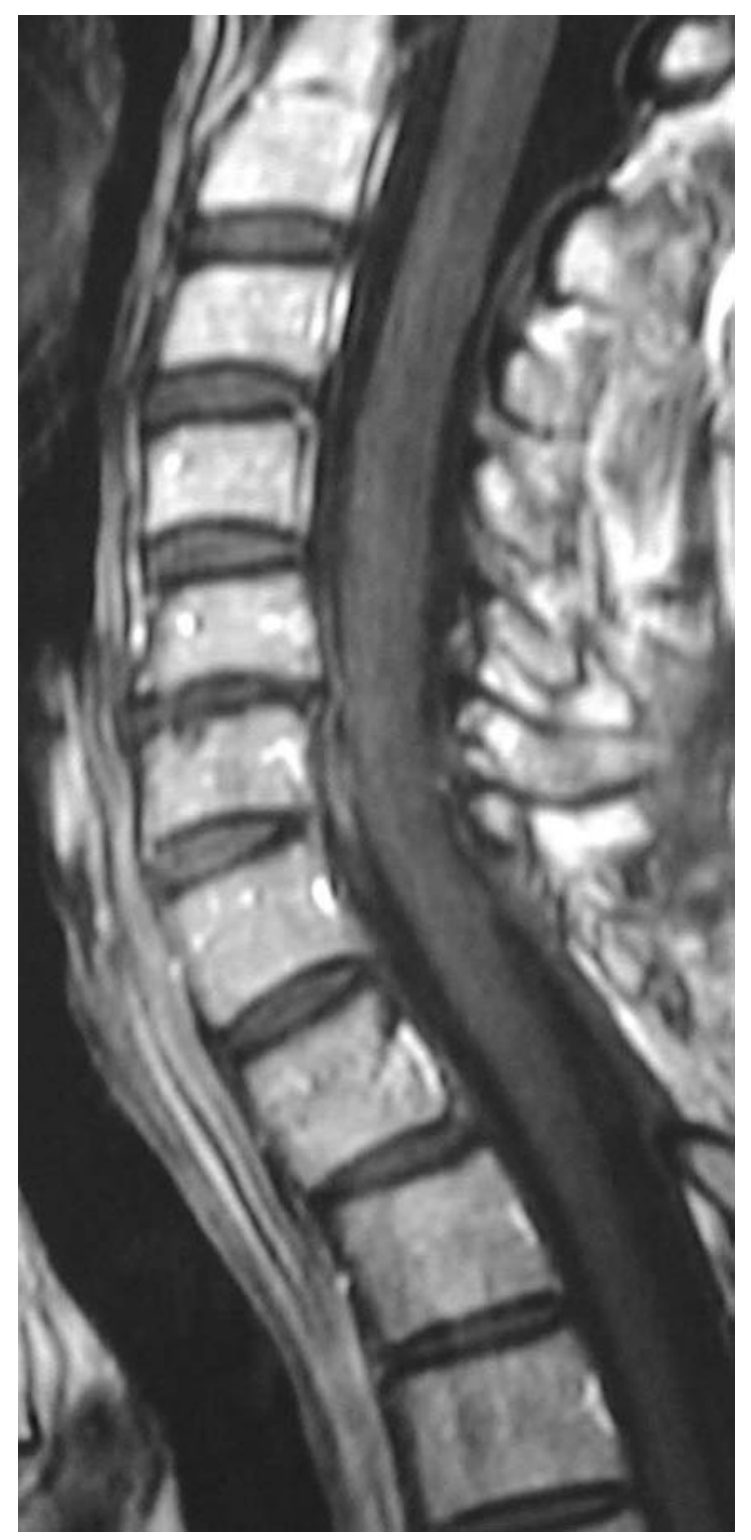

Figura 5. RM plano sagital que muestra la resección completa del componente intrarraquideo del cavernoma.

congénito o adquirido. Se pueden presentar como lesiones únicas o múltiples, y como casos esporádicos o familiares, siendo la localización intracraneal mucho más frecuente que la raquimedular $3,10,17,22,25$. Histológicamente están formados por múltiples vasos sinusoidales de distintos tamaños, compactados y sin tejido neural o glial interpuesto. La pared de estos canales vasculares se compone de un tejido fibrohialino tapizado por una sola capa de células endoteliales, y está desprovista de capa elástica y de músculo liso ${ }^{23,25,28}$. Aunque todos los cavernomas tienen idénticas características anatomopatológicas, incluidas las de tipo inmunohistoquímico y de microscopía electrónica, es habitual en los cavernomas intraparenquimatosos la presen- 
cia de macrófagos con hemosiderina en la periferia ${ }^{3,7,25}$ que no suelen aparecer en los cavernomas epidurales o de los senos venosos intracraneales. Existen además llamativas diferencias macroscópicas según su localización. De esta forma, los cavernomas intraparenquimatosos suelen ser poco vascularizados y angiográficamente ocultos, mientras que los cavernomas vertebrales sin y con extensión epidural y de los senos venosos intracraneales se encuentran muy vascularizados, con aferencias arteriales demostrables en la angiografía. Esta variabilidad ha llevado a algunos autores a pensar que se trata de diferentes tipos de lesión, considerando a los cavernomas epidurales como hamartomas mesenquimales del mismo espectro que los lipomas y angiolipomas ${ }^{7,17,28,29,31}$ o como displasias del mesodermo angioblástico en el espectro de los hemangioblastomas ${ }^{23,24}$, pero sin llegar a concretar una teoría etiopatogénica que sea globalmente aceptada.

No se conoce con exactitud el porcentaje de cavernomas raquídeos que se localizan en el espacio epidural, excluidos los hemangiomas de cuerpo vertebral ${ }^{17}$. Alrededor de unos 80 casos de este tipo han sido publicados desde su primera descripción por Globus y Doshay en 192925,28-31. En la revisión de la literatura que se ha realizado, solamente han sido encontrados 8 casos de CRE con morfología en reloj de arena, similares al caso que presentamos ${ }^{5,6,12,13,15,18,20,21}$. Los CRE, que se ubican preferentemente en el raquis dorsal ${ }^{2,13,17,22,24,25}$, suelen ser lesiones bien circunscritas, lobuladas y encapsuladas, y de un color pardo-rojizo que refleja su naturaleza vascular ${ }^{3,26}$. La presentación clínica es habitualmente en forma de un síndrome lentamente progresivo de compresión medular y/o radicular de diversa gravedad $^{2,27,31}$, aunque se han descrito también síndromes de comienzo agudo (hasta en un $11 \%$ de los casos) secundarios a un incremento brusco del volumen de la lesión por hemorragia o trombosis en su interior ${ }^{7,13,17,19,22,24-26,28}$. Alvarez y col. publicaron el caso de una niña lactante con un síndrome agudo de compresión medular secundario a un hematoma epidural espinal producido por un $\mathrm{CRE}^{1}$ Liu y col. describen el caso de una mujer embarazada que debutó con una paraparesia rápidamente progresiva debida a un CRE torácico. Estos autores especulan con la posibilidad de que se pueda ocasionar un aumento de tamaño de los cavernomas en relación con el embarazo por tres posibles mecanismos: incremento de la presión venosa en vena cava inferior, efecto hormonal directo sobre el endotelio vascular y/o incremento global del volumen sanguíneo?.

El diagnóstico radiológico de los CRE mediante RM muestra unas características inespecíficas, con una señal homogénea isointensa (en relación a la médula) en secuencia potenciada en $\mathrm{T} 1$, y una señal hiperintensa ligeramente menos intensa que el LCR en secuencia potenciada en $\mathrm{T} 2^{2,7,26,31}$. En raras ocasiones se ha descrito la presencia de un halo perilesional con señal hipointensa como mani- festación de depósitos de hemosiderina, similar al observado en los cavernomas intraparenquimatosos ${ }^{8,23,26,28}$; $\sin$ embargo, y a diferencia de estos, los CRE captan de forma intensa y homogénea tras la administración de contraste paramagnético intravenoso $0^{2,7,16,22}$. En este sentido Talachi y col. destacan la similitud de los CRE con los cavernomas desarrollados en otros órganos extraneurales. Según algunos autores, la escasa presencia de hemosiderina en los CRE se explica porque, al no existir barrera hematoencefálica en esta localización, dicho producto de degradación de la hemoglobina sería eliminado con mayor facilidad $d^{7,30,31}$ También en algunos casos se pueden observar zonas de señal hiperintensa en ambas secuencias T1 y T2 debido a hemorragias agudas intralesionales ${ }^{7}$. La TC de columna puede mostrar en ocasiones erosión, remodelación o ensanchamiento del componente óseo foraminal, pero la presencia o no de dicho hallazgo no aporta una especial ayuda para el diagnóstico diferencial ${ }^{3,26,28,31}$. Debido a esta inespecificidad radiológica en la RM y la TC, es frecuente que los CRE sean diagnosticados erróneamente como tumores de la vaina nerviosa (schwanoma o neurofibroma) ${ }^{14,30}$. Otras lesiones con las que se debe realizar un diagnóstico diferencial son linfoma, angiolipoma, meningioma extradural, cordoma, metástasis, y fragmentos discales extruidos y emigrados en el canal raquídeo ${ }^{2,7,8,15,22,26,29-31}$. Hay poca experiencia sobre el interés diagnóstico de la angiografía espinal en los CRE, dado que esta prueba no suele realizarse si no se tiene previamente una alta sospecha diagnóstica con la RM. Excepto en 2 casos $^{3,24}$, la mayoría de los que han sido revisados en la literatura no mostraron un patrón angiográfico de malformación vascular ${ }^{3,17,24,25,28}$. En la paciente que presentamos, se realizó una angiografía selectiva de la lesión que demostró una captación difusa del contraste compatible con tumor hipervascularizado, pero no se llegó a sospechar un posible diagnóstico de cavernoma.

Dada la rareza de los CRE, no se conoce bien su historia natural y es por tanto difícil decidir la actitud a seguir en casos asintomáticos diagnosticados incidentalmente. Para los CRE sintomáticos, el tratamiento de elección es la extirpación quirúrgica ${ }^{3,11,13,17,26,28-31}$. La resección puede ser completa en la gran mayoría de ellos a través de una laminectomía, aunque dependiendo de la extensión de la lesión, es preciso combinar a veces la laminectomía con una toracotomía ${ }^{10}$. En nuestro caso quizás hubiera sido más adecuada un vía de abordaje cervical más lateral o combinada con otra intratorácica para extirpar por completo la lesión, pero dada la benignidad de la misma, se optó por dejar el componente extrarraquídeo y resecarlo en un segundo tiempo en caso de aumentar su tamaño. Mediante técnica microquirúrgica es fácil la extirpación "en bloque" tras liberar la lesión de sus adherencias a la duramadre y a la vaina nerviosa. Para conseguir este objetivo con 
mayor facilidad, se puede intentar disminuir el volumen del cavernoma electrocoagulando su superficie con pinza bipolar $^{28}$. Normalmente, no presentan aferencias arteriales de calibre significativo, y la hemorragia intraoperatoria suele ser mínima ${ }^{28}$. Se desconoce el papel que pueda jugar la embolización en el tratamiento de los CRE, ya que rara vez se dispone de un diagnóstico angiográfico previo a la cirugía. En nuestro caso, pensamos que la embolización prequirúrgica del cavernoma contribuyó de forma importante en la disminución de la hemorragia intraoperatoria. Respecto a la radioterapia, se han publicado algunos casos de administración postoperatoria tras resección incompleta de un cavernoma, y los resultados obtenidos han sido variables y poco concluyentes ${ }^{4,17}$. Debido a los efectos secundarios que puede ocasionar la radioterapia espinal, Tekkök y col. recomiendan para los casos de extirpación subtotal que se realice un seguimiento radiológico de la lesión residual y que, si se observa aumento del tamaño de la misma, se intente un segundo tratamiento quirúrgico ${ }^{29}$. El pronóstico de los CRE tras el tratamiento quirúrgico es bueno en general, aunque depende de la situación neurológica previa del paciente y de la complejidad quirúrgica de cada lesión ${ }^{3,27}$. En los pocos pacientes en los que se ha podido recoger un seguimiento posquirúrgico tras extirpación completa de un CRE, no se han observado recidivas trascurridos periodos de hasta 5 años ${ }^{22,29}$.

Se puede concluir que es muy importante incluir en los diagnósticos diferenciales a aquellas lesiones raras que, por sus especiales características, pudieran ser subsidiarias de tratamientos no quirúrgicos, o modificar la técnica quirúrgica al ser diagnosticadas como hallazgo intraoperatorio inesperado. Los CRE representan un ejemplo de ello en la cirugía raquídea. A pesar de su benignidad histológica, pueden ser potencialmente muy lesivos, y aunque la extirpación quirúrgica parece ser el tratamiento más apropiado, quedan por investigar los resultados de otras técnicas como la embolización endovascular o la radioterapia/ radiocirugía 17,28,29.

\section{Bibliografía}

1. Alvarez, C., Martín Gamero, P., Villarejo, F., Colomar, P., Serrano, A.M., Martínez de Azagra, A.: Paraplejia en lactante por hematoma extradural debido a sangrado de cavernoma espinal. Neurocirugía 1999; 10: 367-371.

2. Aoyagi, N., Kojima, K., Kasai, H.: Review of spinal epidural cavernous hemangioma. Neurol Med Chir (Tokyo) 2003; 43: 471-475.

3. Appiah, G.A., Knuckey, N.W., Robbins P.D.: Extradural spinal cavernous haemangioma: case report and review of the literature. J. Clin Neurosci 2001; 8: 176-179.

4. Ayerbe, J., Lobato, R.D., Domínguez, J., Sánchez, F., Benítez, A.: Hemangioma cavernoso gigante del seno cavernoso: presentación de un caso tratado con irradiación. Neurocirugía 1994; 3: 254-258.

5. Badinand, B., Morel, C., Koop, N., Tran Min, V.A., Cotton, F.: Dumbbell-shaped epidural capillary hemangioma. AJNR Am J Neuroradiol. 2003; 24: 190-192.

6. Bavbek, M., Yurt, A., Caner, H., Altinors, N.: An unusual dumbbell form of cavernous hemangioma of the cervical spine. Kobe J Med Sci 1997; 43: 57-63.

7. Cano, A., Santos, A.L., Romero, A.I., Bravo, F., Delgado, F., Aguilera del Moral, A.: Thoracic spinal epidural hemangioma. Rev Neurol 2001; 33: 195-196.

8. Carlier, R., Engerand, S., Lamer, S., Vallee, C., Bussel, B., Polivka, M.: Foraminal epidural extra osseous cavernous hemangioma of the cervical spine. Spine 2000; 5: 629-631.

9. Chien-lin, L., Dah-jung, Y.: Paraplegia due to vertebral hemangioma during pregnancy. A case report. Spine 1988; 52: 198-203.

10. D'Andrea, G., Ramundo, O.E., Trillo, E., Roperto, R., Isidori, A., Ferrante, L.: Dorsal foramenal extraosseous epidural cavernous hemangioma. Neurosurg Rev 2003; 26 : 292-296.

11. Falch-Tamimi, A., Tamimi, S.O.: Extensive epidural hemangioma with skin and bone involvement: case report. Spine 1995; 20: 2470-2472.

12. Franz, K., Lesoin, F., Leys, D., Krivosic, I., Jomin, M.: Spinal epidural dumbbell-shaped cavernous angioma. Rev Neurol (Paris) 1987; 143: 298-300.

13. Fukushima, M., Nabeshima, Y., Shimazaki, K., Hirohata, K.: Dumbbell-shaped spinal extradural hemangioma. Arch Orthop Trauma Surg 1987; 106: 394-396.

14. Goyal, A., Singh, A.K., Gupta, V., Tatke, M.: Spinal epidural cavernous haemangioma: a case report and review of literature. Spinal Cord 2002; 40: 200-202.

15. Haimes, A.B., Krol, G.: Dumbbell-shaped spinal cavernous hemangioma: a case report. AJNR Am Neuroradiol 1991; 12: 1021:1022.

16. Harrington, J.F., Khan, A., Grunnet, M.: Spinal epidural cavernous angioma presenting as a lumbar radiculopathy with analysis of magnetic resonance imagin characteristics: case report. Neurosurgery 1995; 36: 581-584.

17. Harrison, M.J., Eisenberg, M.B., Ullman, J.S., Oppenheim, J.S., Camins, M.B., Post, K.D.: Symtomatic cavernous malformations affecting the spine and spinal cord. Neurosurgery 1995; 37: 195-205.

18. Honda, E., Hayashi, T., Tokunaga, T., Honda, N., Utsunomiya, H., Sato, Y., et al: The dumbbell shaped vertebral hemangioma developed in the pleural cavity and spinal canal. No Shinkei Geka 1987; 15: 1125-1130.

19. Isla, A., Alvarez, F., Morales, C., García Blazquez, M.: Spinal epidural hemangiomas. J Neurosurg Sci 1993; 37: 3942.

20. Lanotte, M., Massaro, F., Faccani, G., Forni, M., Valentini, M.C.: Dumbbell-shaped epidural cavernous angioma. 
Case report. Ital J Neurol Sci 1994; 15: 429-432.

21. Morioka, T., Nagaki, H., Matsushima, T., Hasuo, K.: Dumbbell-shaped spinal epidural cavernous angioma. Surg Neurol 1986; 25: 142-144.

22. Nguyen, H. M.: Cervicothoracic spinal epidural cavernous hemangioma: a case report and review of the literature. Surg Neurol 2005; 64: 83-85.

23. Padolecchia, R., Acerbi, G., Puglioli, M., Collavoli, P.L., Ravelli, V., Caciagli, P.: Epidural spinal cavernous hemangioma. Spine 1998; 23: 1136-1140.

24. Padovani, R., Tognetti, F., Proietti, D., Pozzati, E., Servadei, F. : Extrathecal Cavernous hemangioma. Surg Neurol 1982; 18: 463-465.

25. Saringer, W., Nöbauer, I., Haberler, C., Ungersböck, K.: Extraforaminal, thoracic, epidural cavernous haemangioma: case report with analysis of Magnetic Resonance Imagin characteristics and review of the literature. Acta Neurochir (Wien) 2001; 143: 1293-1297.

26. Shin, J.H., Lee, H.K., Rhim, S.C., Park, S.H., Choi, C.G., Suh, D.C.: Spinal epidural cavernous hemangioma: MR findings. J Comput Assist Tomogr 2001; 25: 257-261.

27. Singh, R.V., Suys, S., Campbell, D.A., Broome, J.C.: Spinal extradural cavernous angioma. Br J Neurosurg 1993; 7 : 79-81.
28. Talacchi, A., Spinnato, S., Alessandrini, F., Iuzzolino, P., Bricolo, A.: Radiologic and surgical aspects of pure spinal epidural cavernous angiomas. Report on 5 cases and review of the literature. Surg Neurol 1999; 52: 198-203.

29. Tekkök, I.H., Akpmar, G., Güngen, Y.: Extradural lumbosacral cavernous hemangioma. Eur Spine J 2004; 13: 469-473.

30. Thome, C., Zevgaridis, D., Matejic, D., Sommer, C., Krauss, J.K.: Juxtaposition of an epidural intraforaminal cavernous hemangioma and intradural schwannoma. Spine 2004; 29: E524-527.

31. Zevgaridis, D., Büttner, A., Weis, S., Hamburger, G., Reulen, H.J.: Spinal epidural cavernous hemangiomas. Report of three cases and review of the literature. J Neurosurg 1998; 88: 903-908.

Iglesias, S.; Ayerbe, J.; Sarasa, J.L.; Sousa, P.; Torres, C.; Ruiz-Barnés, P.: Cavernoma raquídeo epidural en reloj de arena. Presentación de un caso y revisión de la literatura. Neurocirugía 2008; 19: 248-253.

Correspondencia postal: Dra. Iglesias Moroño. Servicio de Neurocirugía. Fundación Jiménez Díaz. Avda. Reyes Católicos, 2. 28040 Madrid. 